\title{
Pseudoaneurisma post-trasplante renal: a propósito de un caso
}

\section{Pseudoaneurysm, a Complication after Renal Transplantation: A Case Report}

\author{
Candelaria Tregea ${ }^{1}$ Nicolás Roccatagliata ${ }^{1}$ Mariángela Paba ${ }^{1}$ Esteban Jeanmaire ${ }^{1}$ \\ Diego Herrera Vegas ${ }^{2,3}$ Nicolás Sánchez ${ }^{3}$ Roberto Salgado ${ }^{3,4}$ Nebil Larranaga ${ }^{1}$
}

${ }^{1}$ Servicio de Diagnóstico por imágenes, CEMIC, Ciudad autónoma de Buenos Aires, Argentina

2 Servicio de Cirugía vascular, CEMIC, Ciudad autónoma de Buenos Aires, Argentina

${ }^{3}$ Unidad de Trasplante Renal y Renopancreático, Servicio de Nefrología, CEMIC, Ciudad autónoma de Buenos Aires, Argentina

${ }^{4}$ Servicio de Cirugía General, CEMIC, Ciudad autónoma de Buenos Aires, Argentina

Rev Argent Radiol 2021;85:109-112.

Estimados editores,

Los pseudoaneurismas (PSA) secundarios a trasplante renal son patologías poco frecuentes y aquellos de localización extrarrenal, excepcionales (menor al $1 \%){ }^{1-3}$ Las múltiples variantes que poseen en su forma de presentación clínica nos dan la pauta de que deben ser sospechados en todos los pacientes trasplantados, aún aquellos asintomáticos, dada su alta morbi-mortalidad. Por dicho motivo, la detección temprana juega un papel esencial en esa patología, siendo el diagnóstico por imágenes y el intervencionismo los actores principales.

El objetivo de este manuscrito consiste en presentar un caso de PSA extrarrenal como complicación infrecuente en pacientes postrasplante renal.

Presentamos el caso de una paciente femenina de 42 años de edad con trasplante renopancreático por nefropatía diabética. Pasados siete años de la cirugía, presentó signos de rechazo y pielonefritis con consecuente fallo renal y nefrectomía del injerto. Por esa causa, se realizó un nuevo trasplante con anastomosis término lateral de vasos renales a vena y arteria ilíaca externa del lado izquierdo.

Generalmente, el injerto renal es trasplantado en la región extraperitoneal de la fosa ilíaca derecha debido a que la vena

recibido

12 de noviembre de 2019

aceptado

30 de octubre de 2020
Address for correspondence Candelaria Tregea, MD, Riobamba 950, 6Y, Ciudad Autónoma de Buenos Aires, Argentina (e-mail: candetregea@gmail.com).

ilíaca homolateral corre más superficial y horizontalmente, facilitando la anastomosis vascular. ${ }^{4}$ En pacientes con trasplante pancreático, injertos renales previos 0 alteraciones vasculares, se elige la fosa ilíaca izquierda, como en nuestro caso. ${ }^{5}$

A las 72 horas de la cirugía, presentó secreción serosa por herida y evolución tórpida, por lo que se realizó ecografía Doppler (ECOD), observando adyacente al hilio del riñón trasplantado, imagen hipoecoica con flujo Doppler bidireccional (-Fig.1) e imagen líquida que se extiende al tejido celular subcutáneo adyacente. Se solicitó tomografía computada multidetector (TCMD) con contraste endovenoso, donde se observó imagen sacular que realza en fase arterial con intensificación en la fase venosa, de aproximadamente $61 \mathrm{~mm}$, con una luz de $40 \mathrm{~mm}$ (-Fig. 2), la cual impresiona tener origen en la arteria renal a $10 \mathrm{~mm}$ de la anastomosis ilíaca (-Fig. 3) y abundante cantidad de líquido rodeando la misma, que se extiende a la cicatriz. Se decidió realizar angiografía (AG), observando flujo de sangrado activo en trayecto medio de la arteria renal del riñón trasplantado interpretado como PSA (-Fig. 4). En el mismo acto, se realizó angioplastia selectiva con stent cubierto y se obtuvieron resultados exitosos.



(C) 2021. Asociación Civil Sociedad Argentina de Radiología and Federacion Argentina de Asociaciones de Radiología, Diagnóstico por Imágenes y Terapia Radiante. All rights reserved.

This is an open access article published by Thieme under the terms of the Creative Commons Attribution-NonDerivative-NonCommercial-License, permitting copying and reproduction so long as the original work is given appropriate credit. Contents may not be used for commercial purposes, or adapted, remixed, transformed or built upon. (https://creativecommons.org/ licenses/by-nc-nd/4.0/)

Thieme Revinter Publicações Ltda., Rua do Matoso 170, Rio de Janeiro, RJ, CEP 20270-135, Brazil 


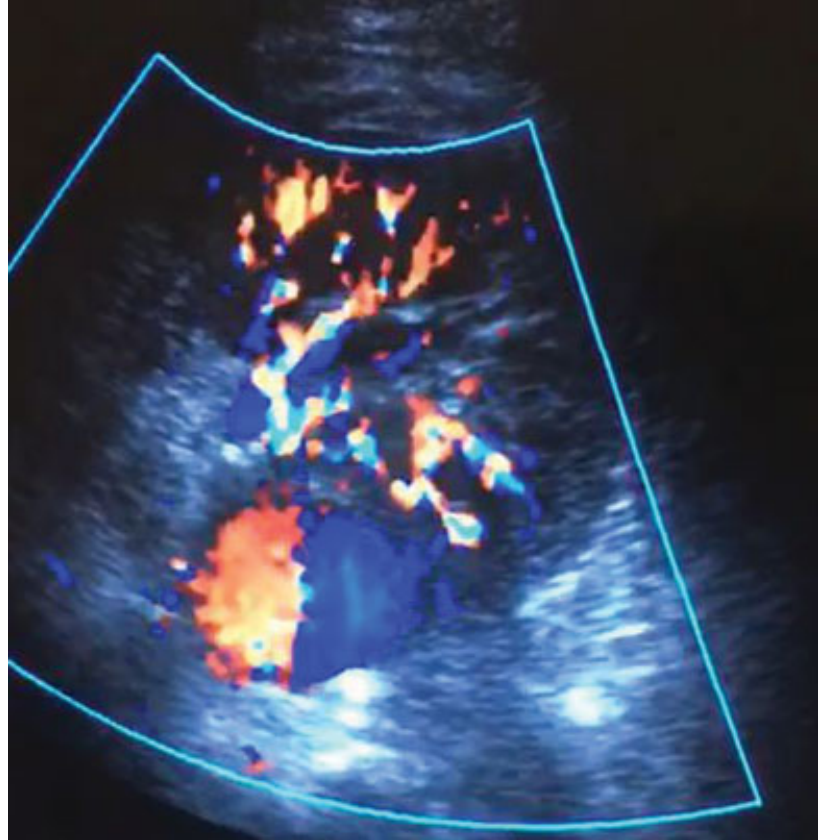

Fig. 1 Estructura sacular con flujo interno bidireccional conformando el signo del Ying-Yang adyacente al hilio del injerto renal, compatible con PSA.

Los PSA surgen en las primeras semanas del postoperatorio y corresponden a una lesión de la pared arterial con la formación de un saco contenido por las capas media o adventicia 0 el tejido conectivo circundante en comunicación con la luz arterial. ${ }^{6}$ Ellos pueden ubicarse de forma intrarrenal o extrarrenal, siendo esos últimos más raros, con una incidencia menor al $1 \%$ y de peor pronóstico. ${ }^{1-3} \mathrm{La}$ etiología de los PSA extrarrenales aún es poco clara; una de las teorías propuestas es la falla en la anastomosis arterial ya que, en esos casos, no intervienen factores degenerativos. ${ }^{1}$ Como diagnóstico diferencial, no se debe descartar la posibilidad de

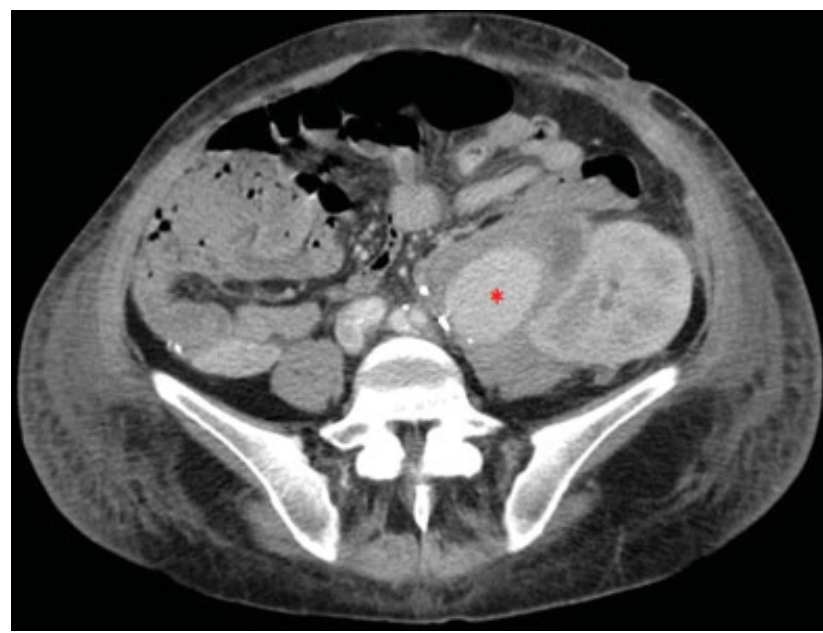

Fig. 2 TCMD con i.v, corte axial. Imagen sacular de $61 \mathrm{~mm}$ de longitud, con realce similar a las estructuras vasculares adyacentes, tras la administración de contraste endovenoso, adyacente al trasplante renal, correspondiente a pseudoaneurisma (asterisco) y líquido libre circundante.

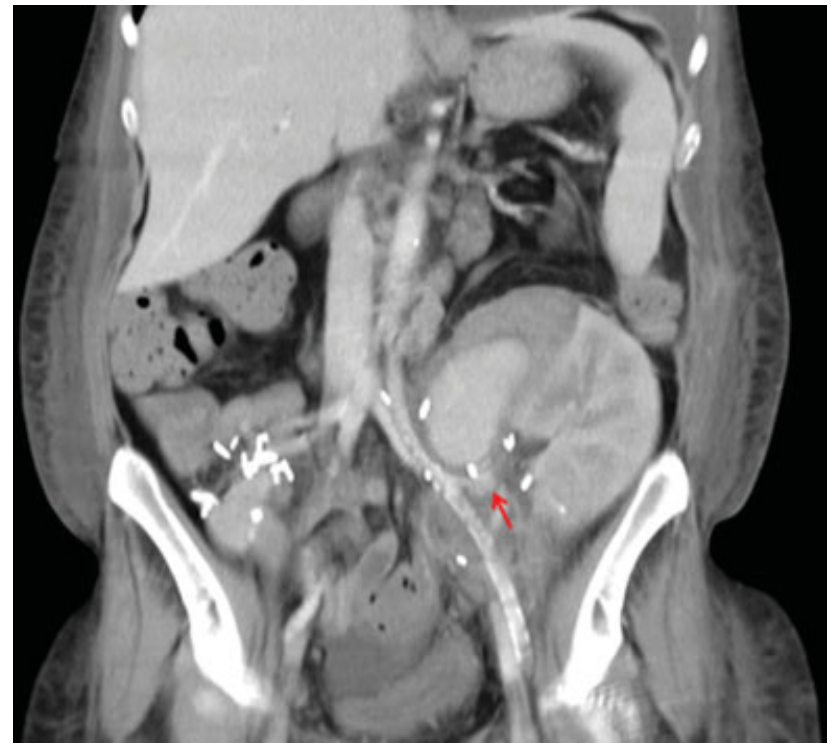

Fig. 3 TCMD con contraste intravenoso (i.v.), corte coronal. Pseudoaneurisma adyacente al trasplante renal, en topografía de la anastomosis con arteria ilíaca externa (flecha).

los aneurismas micóticos, los cuales, a diferencia de los primeros, podrán encontrarse en cualquier sitio de la vasculatura, no únicamente en el sitio de anastomosis. ${ }^{2}$

La clínica puede variar desde formas asintomáticas hasta presentaciones como hipertensión arterial, compresión de estructuras adyacentes, anemia, alteración de la función renal o incluso debutar con hemorragia masiva, que puede tener como consecuencia la pérdida del trasplante y/o la muerte del paciente. ${ }^{3}$

La ECOD, la gammagrafía, la TCMD con contraste endovenoso y la AG son los principales métodos

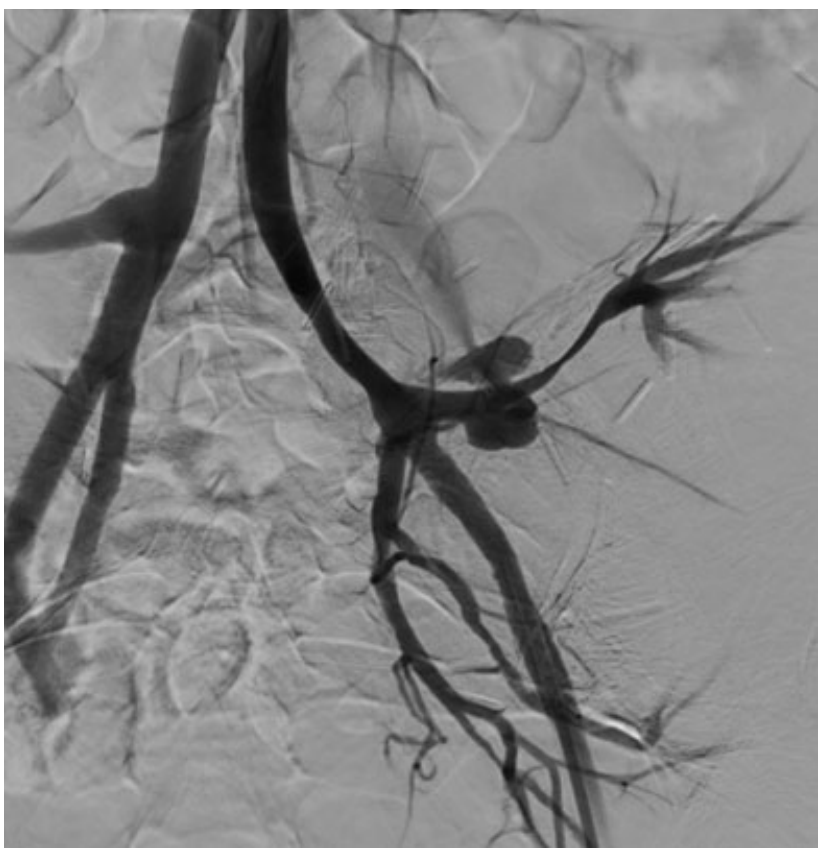

Fig. 4 Flujo de sangrado activo en trayecto medio de la arteria renal del riñón trasplantado interpretado como PSA. 
diagnósticos. ${ }^{1}$ La ECOD muestra flujo sanguíneo dentro de una estructura quística caracterizado por un movimiento en remolino, conformando el típico signo del Ying-Yang. ${ }^{6}$ Sin embargo, tiene como desventaja ser operador dependiente y no tener buen acceso a estructuras profundas como los sitios de anastomosis vasculares o puede ser dificultosa la evaluación por interposición de gas intestinal. ${ }^{2}$ La TCMD da la ventaja de no ser operador dependiente y de obtener imágenes de todas las estructuras involucradas. Así, proporciona herramientas anatómicas para la caracterización de la lesión y la pronta planificación del tratamiento. La AG no solo permite caracterizar de manera puntual el sitio de la lesión, sino que también, en los pacientes hemodinámicamente estables, proporciona una inmediata reparación endovascular, método de tratamiento que es actualmente exitoso y propuesto como abordaje de primera elección para esa patología. ${ }^{2,7}$ Por lo tanto, la TCMD y la AG son dos métodos complementarios que, juntos, son suficientes para la planificación, tratamiento y determinación de su pronóstico.

\section{Responsabilidades éticas}

Protección de personas y animales. Los autores declaran que para esta investigación no se han realizado experimentos en seres humanos ni en animales.

Confidencialidad de los datos. Los autores declaran que han seguido los protocolos de su centro de trabajo sobre la publicación de datos de pacientes.

Derecho a la privacidad y consentimiento informado. Los autores declaran que en este artículo no aparecen datos de pacientes.
Conflicto de Intereses

Los autores declaran no tener ningún conflicto de intereses.

\section{Bibliografía}

1 García de Jalón Martínez A, Pascual Regueiro D, Trívez Boned M.A, Sancho Serrano C, Mallén Mateo E, Gil Martínez P, et al. [Kidney transplantation. Technique and complications]. Actas Urol Esp. 2003;27(09):662-677

2 Fananapazir G, Hannsun G, Wright LA, Corwin MT, Troppmann C. Diagnosis and Management of Transplanted Kidney Extrarenal Pseudoaneurysms: A Series of Four Cases and a Review of the Literature. Cardiovasc Intervent Radiol. 2016;39(11):1649-1653

3 Bracale UM, Carbone F, del Guercio L, Viola D, D'Armiento FP, Maurea S, et al. External iliac artery pseudoaneurysm complicating renal transplantation. Interact Cardiovasc Thorac Surg. 2009;8(06):654-660

4 Kobayashi K, Censullo ML, Rossman LL, Kyriakides PN, Kahan BD, Cohen AM. Interventional radiologic management of renal transplant dysfunction: indications, limitations, and technical considerations. Radiographics. 2007;27(04):1109-1130

5 Sezer TO, Hoscoskun C. Vascular complications after renal transplantation. In: Yamanouchi D, ed. Vascular Surgery. Rijeka: IntechOpen; 2012:85-96. Disponible en: http://www. intechopen.com/books/vascular-surgery/vascular-complicationsafter-renal-transplantation

6 Saad NE, Saad WE, Davies MG, Waldman DL, Fultz PJ, Rubens DJ. Pseudoaneurysms and the role of minimally invasive techniques in their management. Radiographics. 2005;25(1, Suppl 1): S173-S189

7 Akgul E, Binokay F, Aikimbaev K, Aksungur EH. Extrarenal pseudoaneurysm of the arterial anastomosis in a renal transplant: endovascular coil embolization with balloon remodeling technique. Ren Fail. 2011;33(04):452-455 